\title{
Local Origin of Global Contact Numbers in Frictional Ellipsoid Packings
}

\author{
Fabian M. Schaller, ${ }^{1,2, *}$ Max Neudecker, ${ }^{2}$ Mohammad Saadatfar, ${ }^{3}$ Gary W. Delaney, \\ Gerd E. Schröder-Turk, ${ }^{5,1, \dagger}$ and Matthias Schröter ${ }^{2, \$}$ \\ ${ }^{1}$ Institut für Theoretische Physik, Friedrich-Alexander-Universität Erlangen-Nürnberg, 91058 Erlangen, Germany \\ ${ }^{2}$ Max Planck Institute for Dynamics and Self-Organization (MPIDS), 37077 Goettingen, Germany \\ ${ }^{3}$ Applied Maths, RSPhysSE, The Australian National University, Canberra, ACT 0200, Australia \\ ${ }^{4}$ CSIRO Mathematics, Informatics and Statistics, Clayton South, Victoria 3168, Australia \\ ${ }^{5}$ Murdoch University, School of Engineering and IT, Mathematics and Statistics, Murdoch, Western Australia 6150, Australia
}

(Received 7 July 2014; revised manuscript received 3 November 2014; published 14 April 2015)

\begin{abstract}
In particulate soft matter systems the average number of contacts $Z$ of a particle is an important predictor of the mechanical properties of the system. Using x-ray tomography, we analyze packings of frictional, oblate ellipsoids of various aspect ratios $\alpha$, prepared at different global volume fractions $\phi_{g}$. We find that $Z$ is a monotonically increasing function of $\phi_{g}$ for all $\alpha$. We demonstrate that this functional dependence can be explained by a local analysis where each particle is described by its local volume fraction $\phi_{l}$ computed from a Voronoi tessellation. $Z$ can be expressed as an integral over all values of $\phi_{l}$ : $Z\left(\phi_{g}, \alpha, X\right)=\int Z_{l}\left(\phi_{l}, \alpha, X\right) P\left(\phi_{l} \mid \phi_{g}\right) d \phi_{l}$. The local contact number function $Z_{l}\left(\phi_{l}, \alpha, X\right)$ describes the relevant physics in term of locally defined variables only, including possible higher order terms $X$. The conditional probability $P\left(\phi_{l} \mid \phi_{g}\right)$ to find a specific value of $\phi_{l}$ given a global packing fraction $\phi_{g}$ is found to be independent of $\alpha$ and $X$. Our results demonstrate that for frictional particles a local approach is not only a theoretical requirement but also feasible.
\end{abstract}

The average number of contacts $Z$ that a particle forms with its neighbors is the basic control parameter in the theory of particulate systems known as the jamming paradigm [1,2], where $Z$ is a function of the difference between the global volume fraction $\phi_{g}$ and some critical value $\phi_{J}$. For soft, frictionless spheres (a practical example would be an emulsion) this is indeed a good description [3] because additional contacts are formed by the globally isotropic compression of the particles which also increases $\phi_{g}$. However, in frictional granular media such as sand, salt, or sugar the control of $\phi_{g}$ is not achieved by compression but by changing the geometric structure of the sample; if we want to fill more grains into a storage container we do not compress them with a piston, but we tap the container a couple of times on the counter top.

But if $Z$ and $\phi_{g}$ are not simultaneously controlled by a globally defined parameter such as pressure, the idea of a function $Z\left(\phi_{g}\right)$ runs into an epistemological problem: contacts are formed at the scale of individual particles and their neighbors. At this scale the global $\phi_{g}$ is not only undefined, it would even be impossible for a particle scale demon to compute $\phi_{g}$ by averaging over the volume of the neighboring particles. The spatial correlations between Voronoi volumes [4-6] would require it to gather information from a significantly larger volume than the direct neighbors.

To date, only two theoretical approaches have studied $Z$ from a local perspective: Song et al. [7] used a mean-field ansatz to derive a functional dependence between $Z$ and the
Voronoi volume of a sphere. This ansatz has recently been expanded to arbitrary shapes composed of the unions and intersections of frictionless spheres [8,9]. Second, Clusel et al. $[10,11]$ developed the granocentric model which predicts the probability distribution of contacts in jammed, polydisperse emulsions. The applicability of the granocentric model to frictional discs has been shown in [12].

The aim of this experimental study is to go beyond the case of spheres and understand how the average $Z$ in packings of frictional ellipsoids originates from the local physics at the grain level. We find that, to a first approximation, the number of contacts an individual particle forms depends on only two parameters: the material parameter $\alpha$ which is the length ratio between the short and the two (identical) long axes of the ellipsoids. And a parameter that characterizes the cage formed by all the neighboring particles: the local volume fraction $\phi_{l}$ which is the particle volume divided by the volume of its Voronoi cell.

Frictional ellipsoids used in experiments [13-16] exhibit a number of differences to the frictionless ellipsoids often studied numerically [17-23]. The latter have been found to form packings with less than the number of contacts required for isostaticity, which is defined as having enough constraints to block all degrees of freedom of the particles $[17,21,24]$. This apparent paradox has been resolved by Donev et al. [25], who showed that in this analysis the contacts cannot be treated as the contacts between frictionless spheres: the curvature of the ellipsoids blocks rotational degrees of freedom even in the absence of friction. 
TABLE I. Material properties of the particles. The first column displays the color code used in Figs. 2-4. Error bars on $\mu_{s}$ are standard deviations over 15 experiments. The last three columns show the empirical fit parameters for $Z_{l}\left(\phi_{l}, \alpha\right)$ according to Eq. (2).

\begin{tabular}{|c|c|c|c|c|c|c|c|c|c|}
\hline \multirow[b]{2}{*}{$\begin{array}{l}\text { Aspect } \\
\text { ratio } \alpha\end{array}$} & \multicolumn{2}{|c|}{ Half axis } & \multirow[b]{2}{*}{ Type } & \multirow[b]{2}{*}{$\begin{array}{c}\text { Friction } \\
\text { coefficient } \mu_{s}\end{array}$} & \multirow[b]{2}{*}{$\begin{array}{l}\text { Particles in } \\
\text { core region }\end{array}$} & \multirow[b]{2}{*}{$\begin{array}{c}\text { Number of } \\
\text { analyzed packings }\end{array}$} & \multicolumn{3}{|c|}{ Empirical fit parameters for $Z_{l}\left(\phi_{l}, \alpha\right)$} \\
\hline & $\begin{array}{l}\text { Short } \\
{[\mathrm{mm}]}\end{array}$ & $\begin{array}{l}\text { Long } \\
{[\mathrm{mm}]}\end{array}$ & & & & & $a$ & $b$ & $c$ \\
\hline Spheres $\bullet$ & 3.1 & & $3 \mathrm{DP}$ & $0.75 \pm 0.07$ & $660-850$ & 15 & 60.4 & -52.2 & 14.8 \\
\hline $0.80 \bullet$ & 2.65 & 3.30 & $3 \mathrm{DP}$ & $0.75 \pm 0.05$ & $750-850$ & 17 & 60.4 & -52.4 & 15.1 \\
\hline 0.60 & 2.20 & 3.75 & $3 \mathrm{DP}$ & $0.67 \pm 0.03$ & $620-710$ & 16 & 44.7 & -31.0 & 8.4 \\
\hline 0.590 & 2.15 & 3.55 & PPP & $0.38 \pm 0.05$ & $850-910$ & 15 & 63.5 & -53.7 & 15.4 \\
\hline 0.40 & 1.60 & 4.00 & $3 \mathrm{DP}$ & $0.67 \pm 0.05$ & $620-730$ & 10 & 25.3 & -10.7 & 3.9 \\
\hline
\end{tabular}

In contrast, we find packings of frictional ellipsoids to be hyperstatic over the whole range of $\phi_{g}$ studied, in agreement with numerical simulation including friction [26,27].

Particles and preparation.-We study two different types of oblate ellipsoids, the properties of which are summarized in Table I. Figure 1(a) shows pharmaceutical placebo pills (PPP) with $\alpha=0.59$ produced by Weimer Pharma GmbH. Because of their sugar coating, their surface is rather smooth; their static coefficient of friction $\mu_{s}$ against paper is 0.38 (measured using a small sledge on a slowly raised inclined plane). The second particle type displayed in Fig. 1(b) are gypsum ellipsoids cured with resin, produced with a $3 \mathrm{D}$ printer (Zprinter $650, \mathrm{Z}$ corporation). The aspect ratio of these $3 \mathrm{DP}$ particles ranges from 0.4 to 1 (i.e., spherical), their rougher surface results in values of $\mu_{s}$ between 0.67 and 0.75 . Because of the production process, the 3DP particles have hummocks of up to $100 \mu \mathrm{m}$ on their short axis. As a consequence, their volume deviates up to $3 \%$ from a perfect ellipsoid, compared to $1 \%$ for the PPP particles.

Samples are prepared by first creating a loose packing of ellipsoids inside a plexiglass cylinder with an inner diameter of $104 \mathrm{~mm}$; then the samples are tapped in order to increase $\phi_{g}$ to the desired value. We use three different protocols to prepare the initial loose samples-they are indicated by different symbols in the figures below. However, our results do not seem to depend on the initial preparation method, details of which can be found in the

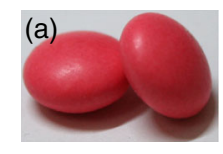

(b)

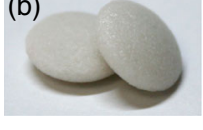

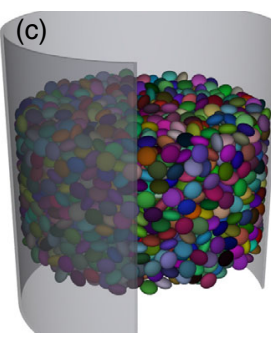

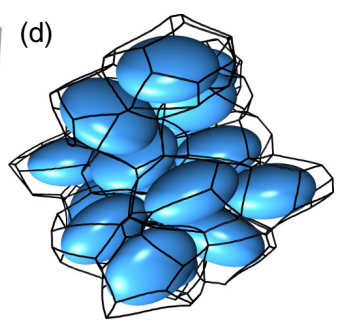

FIG. 1 (color online). (a) Pharmaceutical placebo pills with $\alpha=0.59$. (b) Gypsum particles made with a 3D printer with $\alpha=0.40$. (c) Rendering of the particles detected in a x-ray tomogram. (d) The black wire frame indicates the Voronoi cells of the ellipsoids.
Supplemental Material [28]. Except for the loosest samples, the packings are compactified by applying sinusoidally shaped pulses on an electromagnetic shaker (LDS V555). The width of the pulses is $50 \mathrm{~ms}$ and the peak acceleration $2 g$ (where $g=9.81 \mathrm{~m} / \mathrm{s}^{2}$ ). At a repetition rate of $3 \mathrm{~Hz}$, up to 1500 taps are applied to prepare the highest values of $\phi_{g}$.

Image analysis.-Tomograms of the prepared packings are acquired using $\mathrm{x}$-ray computed tomography (GE Nanotom) with a resolution of $64 \mu \mathrm{m}$ per voxel. The resulting three-dimensional gray scale image is the starting point for the identification of all particle centers and orientations [cf. Fig. 1(c)] using the methods described in Ref. [32]. To reduce boundary effects, only particles with centers that are at least two long axes away from the container walls were included in our analysis; Table I lists the numbers of these core particles. To assure spatial homogeneity, we discard all experiments where the standard deviation of the azimuthally averaged volume fraction is larger than $0.66 \%$. Similarly, to exclude packings with a too large degree of order we only consider samples with $\theta>0.5 \mathrm{rad}$, where $\theta$ is the average angle of the short axis with respect to gravity with $\theta=1$ corresponding to a random orientation (see Supplemental Material [28]). The particle positions and orientations of all experiments reported here can be downloaded from the Dryad repository [33].

From the geometrical representation of the sample we determine the average $Z$ using the contact number scaling method [32]. Finally, the Voronoi cells of the particles are computed with the algorithm described in [34]. Figure 1(d) displays the Voronoi tessellation of a small subset of particles. By dividing the volume of the particle by the volume of the Voronoi cell we obtain for each particle its local volume fraction $\phi_{l}$; the harmonic mean of all particles in the core region corresponds to the global volume fraction $\phi_{g}$.

The average contact number $Z$ as a function of $\phi_{g}$ is displayed in Fig. 2. The main conclusion of Fig. 2 is that the global average of $Z$ depends on both $\phi_{g}$ and $\alpha$. As expected for frictional particles [35-39], the contact number of all samples is significantly above the isostatic value of four [40]. 


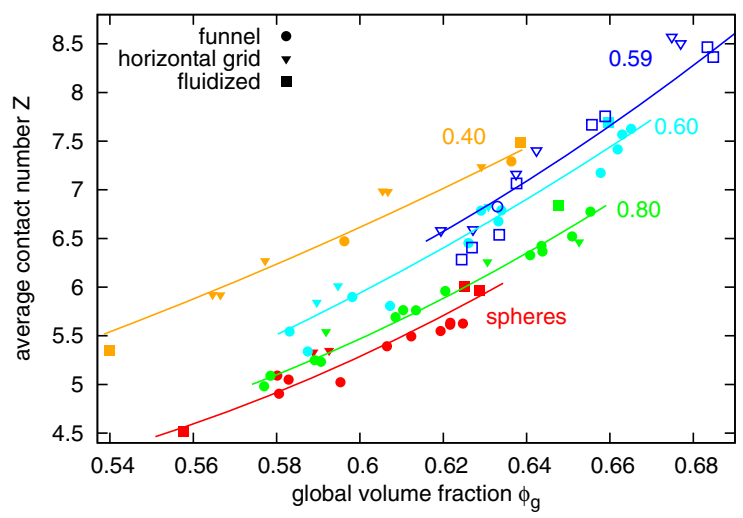

FIG. 2 (color online). Contact number as a function of the global volume fraction. Lines correspond to Eq. (6), which is the numerical integration of the local theory presented here. The different symbols indicate preparation of the initial packing, which is then compactified for all but the loosest samples by tapping. The different aspect ratios and particle types are indicated by different colors; see Table I.

Switching to a local ansatz.-As discussed in the introduction, the formation of contacts between particles needs to be explained solely by parameters which are well defined on the particle level. We therefore start with an ansatz:

$$
Z\left(\phi_{g}, \alpha, X\right)=\int Z_{l}\left(\phi_{l}, \alpha, X\right) P\left(\phi_{l} \mid \phi_{g}, \alpha, X\right) d \phi_{l} .
$$

Here the contact function $Z_{l}\left(\phi_{l}, \alpha, X\right)$ represents the local physics, i.e., the number of contacts formed by a particle of shape $\alpha$, inside a Voronoi cell of size $\phi_{l}$ and potentially characterized by further locally defined variables $X$ such as friction, fabric anisotropy, or measures of local order. $P\left(\phi_{l} \mid \phi_{g}, \alpha, X\right)$ is the conditional probability to find a particle with $\phi_{l}$ in a given packing; an integration over all values of $\phi_{l}$ will result in the global value of $Z$.

In order to measure how $Z_{l}$ depends on $\phi_{l}$, we determine the local contact number for each ellipsoid; see the Supplemental Material [28]. Figure 3(a) shows $Z_{l}\left(\phi_{l}\right)$ for all our experiments. The key observation is that in agreement with our ansatz the curves for the 3DP particles do not depend on the global volume fraction $\phi_{g}$. This result has been previously only shown for spheres [41]. For the PPP particles the collapse is less conclusive, we discuss possible reasons below. In consequence, we take for each value of $\alpha$ the average over all experiments, the resulting $Z_{l}\left(\phi_{l}, \alpha\right)$ curves are shown in Fig. 3(b). Here we have ignored not only $\phi_{g}$ but also all higher-order terms $X$ because within the resolution of our experiments we were not able to discern between different possible candidates. For a discussion of, e.g., $X$ being the orientation of the short axis, see the Supplemental Material [28]. In order to obtain a phenomenological description for $Z_{l}$ we perform for each aspect ratio a parabolic fit using

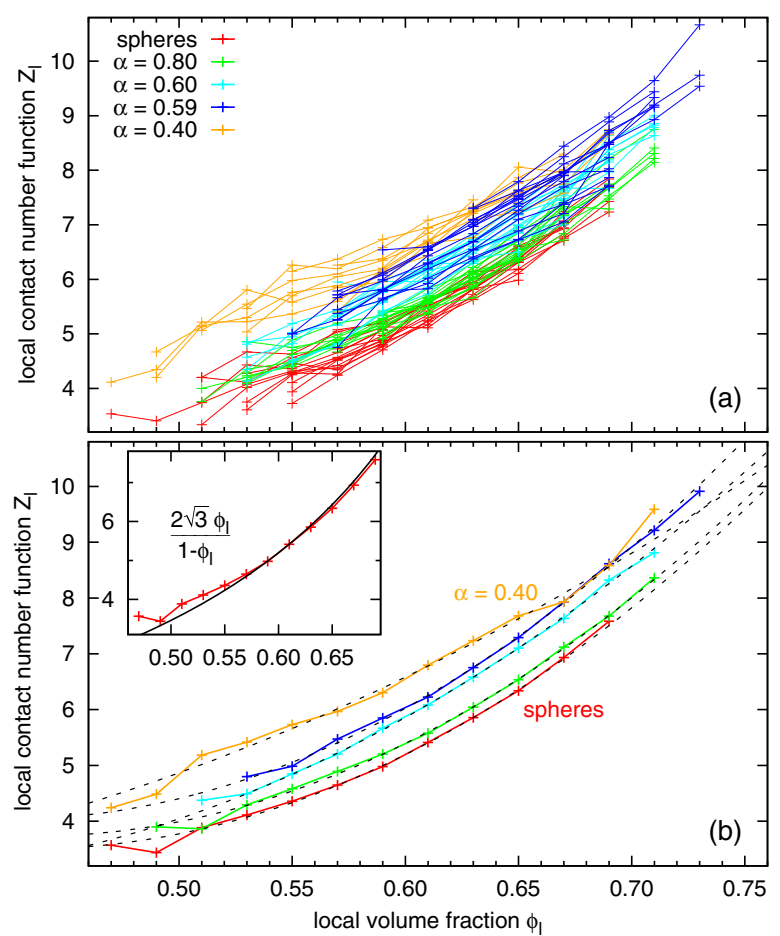

FIG. 3 (color online). Measuring the local contact number function $Z_{l}$ that describes how many contacts an average particle with a local area fraction $\phi_{l}$ will form. In panel (a) each line corresponds to a single experiment, i.e., a single data point in Fig. 2. Each cross represents the average number of contacts a particle with this value of $\phi_{l}$ (using a bin size of 0.02) will form. The colored lines in panel (b) are averages over all data sets (i.e., different values of $\phi_{g}$ ) displayed in the upper panel. The black dashed lines are parabolic fits according to Eq. (2). The inset shows the theoretical result from Song et al. [7] for spheres compared with our sphere data.

$$
Z_{l}\left(\phi_{l}, \alpha\right)=a \phi_{l}^{2}+b \phi_{l}+c .
$$

The results are displayed in Fig. 3(b), the values of the fit parameters $a, b$, and $c$ are listed in Table I.

Fitting Eq. (2) is a purely phenomenological approach, it is justified only by the absence of any theoretical predictions for frictional ellipsoids. The only analytical result available is for spheres [7]; it is in good agreement with our data (without any fit parameters) as shown in the inset of Fig. 3. However, as discussed in the Supplemental Material [28], this result cannot be easily generalized to frictional ellipsoids. Also included in the Supplemental Material are fits which show that even a local reinterpretation of the jamming paradigm does fail to describe the physics.

Properties of the local volume fraction distribution.Figure 4 reveals a number of interesting scaling properties of $P\left(\phi_{l}\right)$. Panel 4(a) shows $P\left(\phi_{l}\right)$ for all different aspect ratio at $\phi_{g} \approx 0.625$. The good agreement indicates that $P\left(\phi_{l}\right)$ is independent of $\alpha$. In Fig. 4(b) a rescaled $P$ is plotted for all values of $\phi_{g}$. This demonstrates, that the mean $\phi_{g}$ and the standard deviation of the local volume fraction distribution 

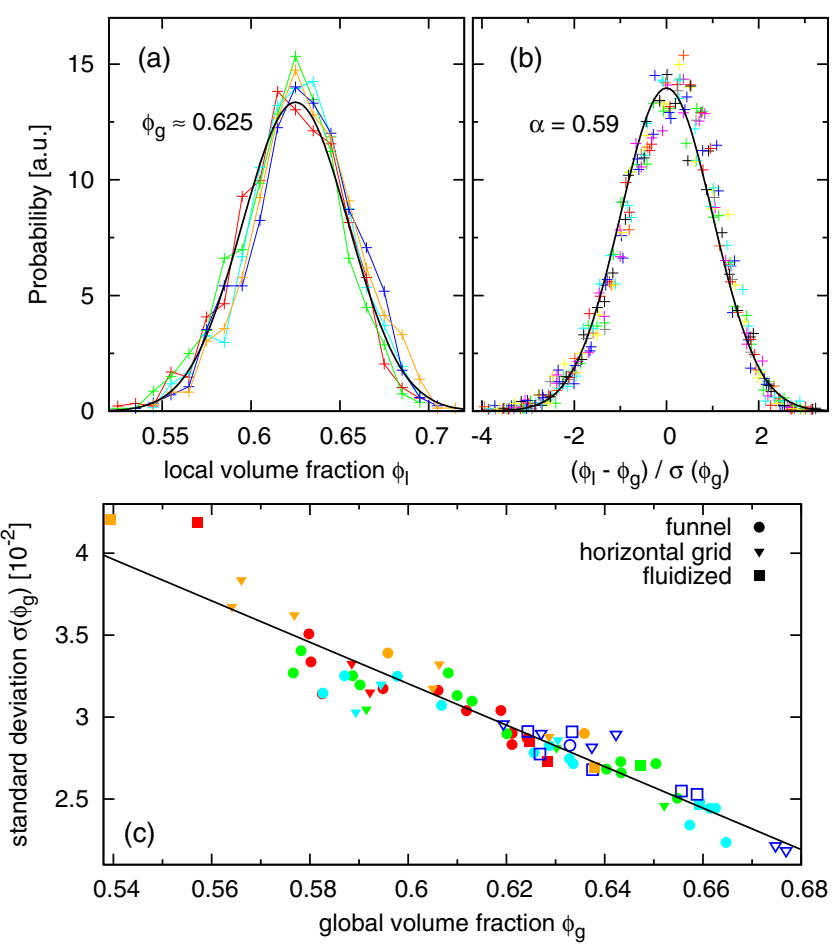

FIG. 4 (color online). Scaling properties of the local volume fraction distribution $P\left(\phi_{l}\right)$. (a) For a given global volume fraction (here $\phi_{g} \approx 0.625$ ) the probability of finding a specific value of $\phi_{l}$ does not depend on $\alpha$. (b) $P$ can be rescaled using $\phi_{g}$ and the standard deviation of the local volume fractions $\sigma$. Shown here are all experiments with $\alpha=0.59$. The black lines in panels (a) and (b) are Gaussian fits using Eq. (4). (c) The standard deviations of the local volume fraction distribution depends only on $\phi_{g}$. Panel includes all experiments shown in Fig. 2. The black line is a linear fit resulting in Eq. (5).

$\sigma\left(\phi_{g}\right)$ are sufficient to describe $P$. This result has previously only been known for spheres [42,43] and discs [12]. Finally, Fig. 4(c) demonstrates that the standard deviation $\sigma\left(\phi_{l}\right)$ of the local packing fraction distribution depends only on $\phi_{g}$ and not $\alpha$.

Together, these results show that $P\left(\phi_{l} \mid \phi_{g}, \alpha, X\right)$ in Eq. (1) can be replaced by $P\left(\phi_{l} \mid \phi_{g}\right)$ :

$$
Z\left(\phi_{g}, \alpha, X\right)=\int Z_{l}\left(\phi_{l}, \alpha, X\right) P\left(\phi_{l} \mid \phi_{g}\right) d \phi_{l}
$$

The advantage of this ansatz is a clear separation of the contact number problem into the local physics at the grain level and a probabilistic term connecting the local and the global volume fraction. Please note that without a better understanding of the origin of the scaling properties shown in Fig. 4 it is not possible to decide on the causality between $\phi_{l}$ and $\phi_{g}$. So writing $P\left(\phi_{l} \mid \phi_{g}\right)$ can imply either that $\phi_{g}$ is the cause of the observed $P\left(\phi_{l}\right)$ or that $\phi_{g}$ can be seen to follow from the prepared $P\left(\phi_{l}\right)$.
In order to get an empirical expression for $Z$ we fit the local packing fraction distribution $P\left(\phi_{l} \mid \phi_{g}\right)$ with a Gaussian [44]

$$
P\left(\phi_{l} \mid \phi_{g}\right)=\frac{1}{\sigma\left(\phi_{g}\right) \sqrt{2 \pi}} e^{\left(-\left(\phi_{l}-\phi_{g}\right)^{2} / 2 \sigma\left(\phi_{g}\right)^{2}\right)}
$$

and the dependence of $\sigma$ on $\phi_{q}$ with a linear equation which yields

$$
\sigma\left(\phi_{g}\right)=-0.126 \phi_{g}+0.109 .
$$

Both fits are displayed as black lines in Fig. 4. Inserting Eqs. (2) and (4) into Eq. (3) and performing the integration leads to

$$
Z\left(\phi_{g}, \alpha\right)=a \sigma\left(\phi_{g}\right)^{2}+a \phi_{g}^{2}+b \phi_{g}+c,
$$

with $\sigma$ according to Eq. (5) and $a, b, c$ as shown in Table I.

A comparison of our experimental data with Eq. (6) is shown in Fig. 2. The good agreement for all 3DP particles demonstrates the validity of our ansatz Eq. (3). For the PPP particles with $\alpha=0.59$ the agreement is only fair, pointing to the need for an additional parameter $X$ in $Z_{l}\left(\phi_{g}, \alpha, X\right)$. However, the experimental scatter does not allow us to assess the type of higher order corrections required. The need for inclusion of such a parameter can also stem from the history-dependent behavior of frictional particles. It has recently been shown for spheres [45] and tetrahedra [39,46] that for identical $\phi_{g}$ the contact number can depend on the preparation history; modeling such behavior will require the addition of further locally defined parameters.

Conclusion.-The global contact numbers of packings of frictional spheres and ellipsoids can be explained by an ansatz that combines a local contact function and a conditional probability. The contact function does depend solely on parameters defined on the particles scale, including the local volume fraction and the aspect ratio of the particles. The conditional probability to find a particle with a specific local volume fraction is sufficiently described by the global volume fraction alone. We expect our results (and our data sets, available as open data [33]), to be a valuable reference point for the generalization of existing theoretical approaches such as the granocentric model $[10,11]$ or the statistical mechanics approach to granular media $[7,8]$ towards frictional granular matter. Extensions of our contact function including other locally defined parameters, such as, e.g., the fabric anisotropy, should be able to describe nonisotropic effects, such as observed in shearjammed frictional packings $[47,48]$.

We thank Weimer Pharma $\mathrm{GmbH}$ for providing the PPP samples, Martin Brinkmann, Stephan Herminghaus, Marco Mazza, Klaus Mecke, and Jean-François Métayer for valuable discussions and Matthias Hoffmann for 
programming support. We acknowledge funding by the German Science Foundation (DFG) through the research group "Geometry and Physics of Spatial Random Systems" under Grant No. SCHR-1148/3-2.

*fabian.schaller@physik.uni-erlangen.de †g.schroeder-turk@murdoch.edu.au *matthias.schroeter@ds.mpg.de

[1] A. J. Liu and S. R. Nagel, Annu. Rev. Condens. Matter Phys. 1, 347 (2010).

[2] M. van Hecke, J. Phys. Condens. Matter 22, 033101 (2010).

[3] G. Katgert and M. v. Hecke, Europhys. Lett. 92, 34002 (2010).

[4] F. Lechenault, F. d. Cruz, O. Dauchot, and E. Bertin, J. Stat. Mech. (2006) P07009.

[5] S.-C. Zhao, S. Sidle, H. L. Swinney, and M. Schröter, Europhys. Lett. 97, 34004 (2012).

[6] S.-C. Zhao, Ph. D. thesis, Georg-August University, Göttingen, Germany (2013), http://hdl.handle.net/11858/ 00-1735-0000-0015-9D98-C

[7] C. Song, P. Wang, and H. A. Makse, Nature (London) 453, 629 (2008).

[8] A. Baule, R. Mari, L. Bo, L. Portal, and H. A. Makse, Nat. Commun. 4, 2194 (2013).

[9] A. Baule and H. A. Makse, Soft Matter 10, 4423 (2014).

[10] M. Clusel, E. I. Corwin, A. O. N. Siemens, and J. Brujić, Nature (London) 460, 611 (2009).

[11] E. I. Corwin, M. Clusel, A. O. N. Siemens, and J. Brujić, Soft Matter 6, 2949 (2010).

[12] J. G. Puckett, F. Lechenault, and K. E. Daniels, Phys. Rev. E 83, 041301 (2011).

[13] W. Man, A. Donev, F. H. Stillinger, M. T. Sullivan, W. B. Russel, D. Heeger, S. Inati, S. Torquato, and P. M. Chaikin, Phys. Rev. Lett. 94, 198001 (2005).

[14] S. Farhadi and R. P. Behringer, Phys. Rev. Lett. 112, 148301 (2014).

[15] C. Xia, K. Zhu, Y. Cao, H. Sun, B. Kou, and Y. Wang, Soft Matter 10, 990 (2014).

[16] S. Wegner, R. Stannarius, A. Boese, G. Rose, B. Szabó, E. Somfai, and T. Börzsönyi, Soft Matter 10, 5157 (2014).

[17] A. Donev, I. Cisse, D. Sachs, E. A. Variano, F. H. Stillinger, R. Connelly, S. Torquato, and P. M. Chaikin, Science 303, 990 (2004).

[18] A. Donev, F. H. Stillinger, P. M. Chaikin, and S. Torquato, Phys. Rev. Lett. 92, 255506 (2004).

[19] M. Mailman, C. F. Schreck, C. S. O'Hern, and B. Chakraborty, Phys. Rev. Lett. 102, 255501 (2009).

[20] Z. Zeravcic, N. Xu, A. J. Liu, S. R. Nagel, and W. v. Saarloos, Europhys. Lett. 87, 26001 (2009).

[21] C. F. Schreck, N. Xu, and C. S. O'Hern, Soft Matter 6, 2960 (2010).

[22] S. K. Mkhonta, D. Vernon, K. R. Elder, and M. Grant, Europhys. Lett. 101, 56004 (2013).

[23] O. Stenzel, M. Salzer, V. Schmidt, P. W. Cleary, and G. W. Delaney, Granular Matter 16, 457 (2014).

[24] R. M. Baram and P. G. Lind, Phys. Rev. E 85, 041301 (2012).
[25] A. Donev, R. Connelly, F. H. Stillinger, and S. Torquato, Phys. Rev. E 75, 051304 (2007).

[26] R. Guises, J. Xiang, J.-P. Latham, and A. Munjiza, Granular Matter 11, 281 (2009).

[27] G. W. Delaney, J. E. Hilton, and P. W. Cleary, Phys. Rev. E 83, 051305 (2011).

[28] See Supplemental Material at http://link.aps.org/ supplemental/10.1103/PhysRevLett.114.158001, for preparation methods, the contact number algorithm and a comparison with existing local theories. It also contains a discussion of the global orientation angle of the particles and the dependence of the contact number on the area fraction, which includes Refs. [29-31].

[29] D. Frenkel, B. M. Mulder, and J. P. McTague, Phys. Rev. Lett. 52, 287 (1984).

[30] M. Radu, P. Pfleiderer, and T. Schilling, J. Chem. Phys. 131, 164513 (2009).

[31] G. Odriozola, J. Chem. Phys. 136, 134505 (2012).

[32] F. M. Schaller, M. Neudecker, M. Saadatfar, G. Delaney, K. Mecke, G. E. Schröder-Turk, and M. Schröter, AIP Conf. Proc. 1542, 377 (2013).

[33] Dryad, DOI: 10.5061/dryad.rf623.

[34] F. M. Schaller, S. C. Kapfer, M. E. Evans, M. J. Hoffmann, T. Aste, M. Saadatfar, K. Mecke, G. W. Delaney, and G. E. Schröder-Turk, Philos. Mag. 93, 3993 (2013).

[35] L. E. Silbert, D. Ertaş, G. S. Grest, T. C. Halsey, and D. Levine, Phys. Rev. E 65, 031304 (2002).

[36] H. P. Zhang and H. A. Makse, Phys. Rev. E 72, 011301 (2005).

[37] K. Shundyak, M. van Hecke, and W. van Saarloos, Phys. Rev. E 75, 010301 (2007).

[38] S. Henkes, M. van Hecke, and W. van Saarloos, Europhys. Lett. 90, 14003 (2010).

[39] M. Neudecker, S. Ulrich, S. Herminghaus, and M. Schröter, Phys. Rev. Lett. 111, 028001 (2013).

[40] Frictional contacts fix three force components, one normal and two tangential. These are shared between the two particles involved, so per particle each contact provides 1.5 constraints. As each particle possesses 6 degrees of freedom, at minimum four contacts are needed to block all of them.

[41] T. Aste, M. Saadatfar, and T. J. Senden, J. Stat. Mech. (2006) P07010.

[42] T. Aste, T. D. Matteo, M. Saadatfar, T. J. Senden, M. Schröter, and H. L. Swinney, Europhys. Lett. 79, 24003 (2007).

[43] F. W. Starr, S. Sastry, J. F. Douglas, and S. C. Glotzer, Phys. Rev. Lett. 89, 125501 (2002).

[44] We approximate $P\left(\phi_{l} \mid \phi_{g}\right)$ by a Gauss function, as this allows explicit integration of Eq. (2) leading to Eq. (6). Note the differences to the $k$-Gamma distributions [42] for the Voronoi cell volumes of spheres (that require the minimal possible Voronoi volume as an additional parameter, unknown for ellipsoids) are small.

[45] I. Agnolin and J.-N. Roux, Phys. Rev. E 76, 061302 (2007).

[46] N. N. Thyagu, F. M. Schaller, M. Neudecker, and M. Schröter, arXiv:1501.04472.

[47] D. Bi, J. Zhang, B. Chakraborty, and R. P. Behringer, Nature (London) 480, 355 (2011).

[48] M. Grob, C. Heussinger, and A. Zippelius, Phys. Rev. E 89, 050201 (2014). 